binding. The keto group in 4-oxoDHA is close enough to the acidic head group to participate in the electrostatic interactions with helix 12. In contrast, with 6-oxoOTO, the keto group is too far away to form such a hydrogen bond. Interestingly, the 6-oxoOTO also demonstrates reduced activation of the AP2 gene at saturating doses compared to 4-oxoDHA, suggesting that the position of the keto group affects ligand activity.

These studies describe the first structures of PPAR $\gamma$ bound to endogenous and covalently bound ligands ${ }^{7,8}$. They demonstrate that two electrostatic clusters on opposite ends of the ligand binding pocket (Fig. 2c) allow simultaneous binding of two ligands. Furthermore, covalent binding or interaction with the helix 3 electrostatic cluster has an impact on ligand interaction with helix 12, and may define specific activity profiles.
An unresolved issue is whether interaction with the helix 3 cluster is sufficient to modulate activity, as synthetic partial agonists can show graded transcriptional responses without stabilizing helix 12 (ref. 4), suggesting that some other mechanism contributes to receptor activation. The recently solved structure of the full-length PPAR $\gamma$-RXR heterodimer demonstrates that the $\beta$-sheet region of PPAR $\gamma$ binds directly to the RXR DNA binding domain (F. Rastinejad, personal communication). Thus, differential binding of ligands to the $\beta$-sheet and helix 3 region of PPAR $\gamma$ may also affect transcriptional activity through modulation of its interaction with RXR.

1. Kliewer, S.A., Xu, H.E., Lambert, M.H. \& Willson, T.M. Recent Prog. Horm. Res. 56, 239-263 (2001).

2. Lehmann, J.M. et al. J. Biol. Chem. 270, 12953-12956 (1995).
3. Berger, J.P. et al. Mol. Endocrinol. 17, 662-676 (2003).

4. Bruning, J.B. et al. Structure 15, 1258-1271 (2007).

5. Shiraki, T. et al. J. Biol. Chem. 280, 14145-14153 (2005).

6. Schopfer, F.J. et al. Proc. Natl. Acad. Sci. USA 102 2340-2345 (2005).

7. Itoh, T. et al. Nat. Struct. Mol. Biol. 15, 924-931 (2008).

8. Li, Y. et al. Nat. Struct. Mol. Biol. 15, 865-867 (2008).

9. Gearing, K.L., Gottlicher, M., Teboul, M., Widmark, E. \& Gustafsson, J.A. Proc. Natl. Acad. Sci. USA 90 , 1440-1444 (1993).

10. Shulman, A.I. \& Mangelsdorf, D.J. N. Engl. J. Med. 353, 604-615 (2005).

11. Nettles, K.W. \& Greene, G.L. Annu. Rev. Physiol. 67 , 309-333 (2005).

12. Steinmetz, A.C., Renaud, J.P. \& Moras, D. Annu. Rev Biophys. Biomol. Struct. 30, 329-359 (2001).

13. Shiau, A.K. et al. Cell 95, 927-937 (1998).

14. Perissi, V. \& Rosenfeld, M.G. Nat. Rev. Mol. Cell Biol. 6, 542-554 (2005).

15. Xu, H.E. et al. Nature 415, 813-817 (2002).

16. Nolte, R.T. et al. Nature 395, 137-143 (1998).

17. Oberfield, J.L. et al. Proc. Natl. Acad. Sci. USA 96 6102-6106 (1999).

\title{
How does Plasmodium falciparum stick to CSA? Let's see in the crystal
}

\author{
Graham A Bentley \& Benoît Gamain
}

\begin{abstract}
The crystal structure of the CSA-binding Duffy-binding-like domain DBL3x of the VAR2CSA-encoded PfEMP1 adhesin has been solved in the free state and complexed with CSA oligosaccharides, shedding light on the major host-parasite interaction in pregnancy-associated malaria.
\end{abstract}

Pregnancy-associated malaria (PAM) contributes to disease and death in both the primigravida and the developing fetus ${ }^{1}$. In subsequent pregnancies, women develop protective antibodies, which indicates the feasibility of an effective PAM vaccine. Two recent papers may provide a rational basis for the development of such a vaccine ${ }^{2,3}$.

Of the four human-infecting malaria parasites, Plasmodium falciparum causes the most severe forms of the disease. Infection by $P$. falciparum is distinguished by massive sequestration of parasitized erythrocytes by adhesion to the microvasculature in

Graham A. Bentley is at the Institut Pasteur, Unité d'Immunologie Structurale, Centre National de la Recherche Scientifique URA2185, F-75724 Paris Cedex 15, France. Benoît Gamain is at the Institut Pasteur, Unité de Biologie des Interactions Hôte-Parasite, Centre National de la Recherche Scientifique URA2581, F-75724 Paris Cedex 15, France. e-mail:bentley@pasteur.frorbgamain@pasteur.fr different tissues or to other blood cells, forming aggregates that block blood flow ${ }^{4}$. These phenomena, which contribute to different clinical manifestations of malaria, are mediated by adhesins that are expressed by the parasite during the intra-erythrocyte stage of the life cycle and that are collectively called $P$. falciparum erythrocyte membrane protein 1 (PfEMP1) ${ }^{5,6}$. PfEMP1 is a highly polymorphic family of proteins with about 60 members, encoded by the var gene ensemble $^{7}$, that are transported to the surface of parasitized erythrocytes, with their extracellular region exposed for interaction with diverse host receptors ${ }^{4}$. Sequestration of infected erythrocytes in peripheral tissues and organs via PfEMP1 is the parasite's mode of defense against destruction during passage through the spleen, which is able to detect the altered morphology of the parasitized host cell ${ }^{8}$. But this comes at a price, since the exposure of these antigens also renders $P$. falciparum vulnerable to attack by the immune system; hence the polymorphic nature of PfEMP1 has a role in immune evasion. Thus, a balance must be sought between the high polymorphism in PfEMP1 and the preservation of receptor binding specificity.

The extracellular regions of PfEMP1 variants have a modular organization, with varying numbers of Duffy-bindinglike (DBL) and cysteine-rich interdomain regions (CIDRs) ${ }^{6}$. The DBL fold is unique to Plasmodium species and is found as one or more modules in different paralogues that function as adhesins to host cell receptors. Both DBL and CIDR domains are highly polymorphic and can be classified into distinct classes and subclasses according to distinguishing sequence signatures ${ }^{9}$. For some of these classes, specificity for particular host receptors in the vasculature or on blood cells has been established. Because only one PfEMP1 variant is presented on the erythrocyte surface at a time ${ }^{10}$, a given parasite phenotype is usually targeted to a specific host receptor.

Extensive accumulation of parasitized erythrocytes in the placental intervillous blood spaces mediated by adhesion to the 


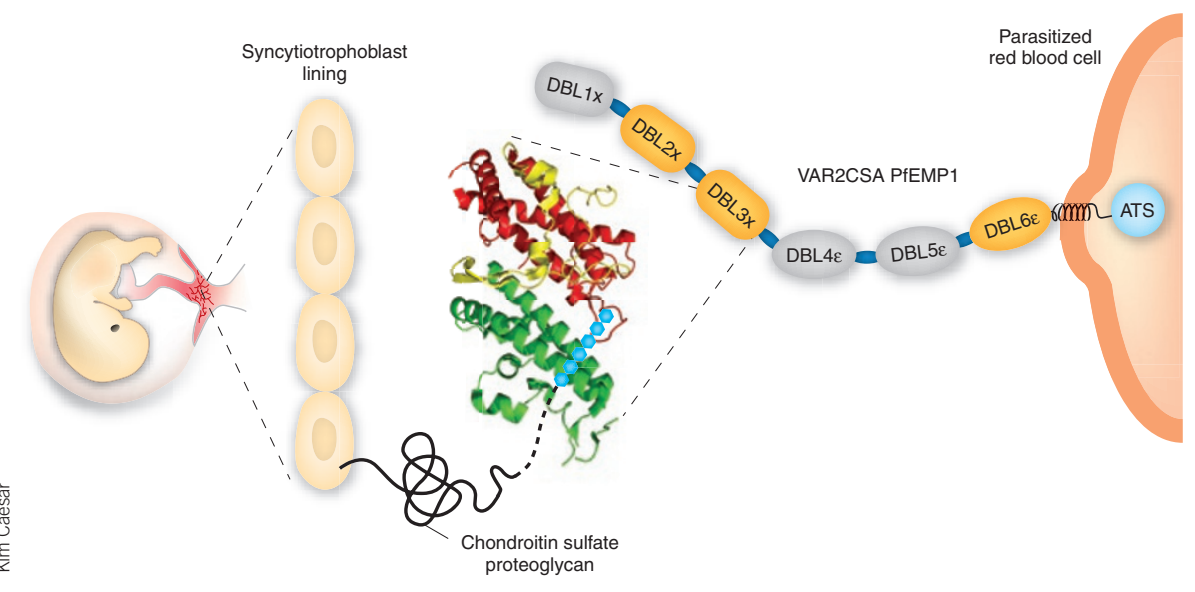

Figure 1 A schematic representation of the VAR2CSA PfEMP1 variant anchored to the membrane of the infected erythrocyte. Clusters of several PfEMP1 molecules occur on protuberances, or 'knobs', which are characteristic surface features of $P$. falciparum-infected erythrocytes. The adhesin comprises a large extracellular $\mathrm{N}$-terminal region with six DBL domains, a single transmembrane segment and a cytoplasmic acid terminal segment (ATS) at the $C$ terminus. The first three DBL domains belong to class $X$, whereas the last three belong to class $\varepsilon$. Individual recombinant DBL domains from VAR2CSA that have been shown to bind CSA are colored in orange. The DBL3X domain, illustrated as an enlargement in ribbon form, is a highly $\alpha$-helical protein (PDB 3CML). The subdomains 1, 2 and 3 are colored yellow, red and green, respectively. VAR2CSA binds to the CSA moiety of placental chondroitin sulfate proteoglycan present on the surface of the syncytiotrophoblast cells in the placenta. The CSA ligand is shown schematically as hexagons (blue), positioned over the region covering subdomains 2 and 3 identified by the two research groups as the CSA binding site.

placental chondroitin-4-sulfate (CSA) occurs in PAM and has been linked to the severe disease outcome ${ }^{11}$. After multiple pregnancies, women acquire protective antibodies that block binding of infected erythrocytes to CSA and cross-react with geographically diverse placental isolates, which suggests that the surface molecules expressed by infected erythrocytes in PAM have conserved epitopes ${ }^{12}$.

Compelling evidence implicates VAR2CSA, a member of the PfEMP1 family, in the direct binding to placental chondroitin sulfate proteoglycans. Indeed, the var2CSA gene is transcriptionally upregulated in both placental isolates and laboratory parasites selected to bind $\mathrm{CSA}^{13}$, and var2CSA-knockout parasites have revealed that no other parasite ligand is able to promote massive adhesion in the placenta $^{14,15}$. Furthermore, the VAR2CSA protein contains multiple CSA-binding domains ${ }^{16}$ and is the target of maternal antibodies ${ }^{17}$. This, and the presence of conserved epitopes among different parasite isolates, makes VAR2CSA the most promising candidate for a PAM vaccine.

VAR2CSA is a $350-\mathrm{kDa}$ protein that carries six DBL domains; among them, three mediate binding to CSA (Fig. 1). The crystal structure of the CSA-binding domain DBL3x from the VAR2CSA PfEMP1 variant of the FCR3CSA laboratory strain has now been determined by two independent groups ${ }^{2,3}$. These two reports provide not only the first structural description of a DBL domain from a PfEMP1 adhesin but also one that is very important for understanding PAM.

The crystal structures of the DBL domain from different Plasmodium adhesins have been determined recently: EBA-175 from P. falciparum $^{18}$ and PkDBP, the Duffybinding protein from P. knowlesi ${ }^{19}$ (from which the DBL domain takes its name). These studies have revealed the DBL motif as a highly $\alpha$-helical structure divided into three subdomains. As expected, the DBL3x structure closely resembles these DBL motifs, with essentially the same arrangement of the helical elements that typify this fold. Despite low conservation of sequence, Higgins ${ }^{2}$ calculates that the root mean square difference between the $\mathrm{C} \alpha$ positions of residues in the common helical regions ranges from $1.5 \AA$ and $2.2 \AA$ when comparing DBL $3 x$ to PkDBP and EBA-175, respectively.

Higgins ${ }^{2}$ and Singh et al. ${ }^{3}$ have identified the same region on DBL3x as the CSA binding site, where a number of positively charged amino acids (mainly lysine) form a contiguous patch of positive charge on the surface of subdomain 3 and, to a lesser extent, subdomain 2 (Fig. 1). Singh et al. ${ }^{3}$ analyzed cocrystals of the DBL3x domain with CSA oligosaccharides of different lengths, ranging up to 14 residues, whereas Higgins $^{2}$ solved a complex formed only with the disaccharide, the basic repeat unit of CSA. In each case, residual electron density in the maps was observed that could not be accounted for by the protein. Frustratingly, this additional density could not be interpreted in terms of molecular structure because the ligand was largely disordered. But this is not unprecedented. For example, a similar disorder in the ligand was observed in the crystal structure of the complex formed between thrombospondin-1 and heparin, another sulfated oligosaccharide ${ }^{20}$. Disorder in the bound oligosaccharide may be a consequence of its source: the CSA used here was of biological origin and is thus not uniformly sulfated along the oligosaccharide chain. Both teams suggest that the DBL3XCSA interaction is essentially ionic. Thus, the disorder may also be a consequence of multiple binding modes due to flexibility in the lysine side chains lining the binding site and/or conformational lability in the CSA chain. Singh et al. ${ }^{3}$ confirmed a binding role for certain lysine residues on subdomain 3 using mutagenesis and subsequent binding studies to CSA-expressing cells and by isothermal titration calorimetry; but additional mutants must be tested to confirm the real extent of the CSA binding site. Higgins ${ }^{2}$ suggests that a polymorphic loop on subdomain 2 may protect some conserved regions of the binding site from antibodies, but this must be reconciled with the fact that some of the conserved lysines implicated in the CSA interaction are exposed on helix $\mathrm{H} 5$ in subdomain 3 and are thus accessible to antibodies. Indeed, this may explain why women exposed to malaria acquire immunity to placental infections with each pregnancy. Thus, one important outcome from these two studies is that the structural data may permit a more rational approach to vaccine development by identifying conformational regions that are conserved between different polymorphic forms of VAR2CSA and that could elicit broadly neutralizing and adhesion-blocking antibodies. For example, a construct based on subdomain 3, which carries a conserved part of the CSA binding site, may be sufficient to induce antibodies that block placental sequestration of potentially all VAR2CSA phenotypes in the field.

These two studies represent a major advance in our appreciation of structure-function relationships in VAR2CSA, but they are only a first step toward a more complete understanding 
of placental sequestration. Two other VAR2CSA domains-DBL2X and DBL6e - bind CSA as well; is there synergy between these ligandbinding domains in the overall function of VAR2CSA? Is there a defined three-dimensional organization of the DBL domains within a PfEMP1 polypeptide chain, and is this important for function? What is the role of DBL4E, which displays very low polymorphism - is it structural only? Does the extracellular region of VAR2CSA have a quarternary organization, either with other VAR2CSA molecules or other proteins on the infected erythrocyte surface? And if so, what role does this have? These questions, of course, apply to PfEMP1 variants specific for other host receptors as well, but the present structural results should inspire us to seek a more general understanding of these interactions, which have a direct role in severe malaria pathologies.

1. Brabin, B.J. et al. Placenta 25, 359-378 (2004).

2. Higgins, M.K. J. Biol. Chem. 283, 21842-21846 (2008).

3. Singh, K. et al. Nat. Struct. Mol. Biol. 15, 932-938 (2008)

4. Miller, L.H., Baruch, D.I., Marsh, K. \& Doumbo, O.K. Nature 415, 673-679 (2002).

5. Baruch, D.I. et al. Cell 82, 77-87 (1995).

6. Su, X.Z. et al. Cell 82, 89-100 (1995).

7. Gardner, M.J. et al. Nature 419, 498-511 (2002).

8. David, P.H., Hommel, M., Miller, L.H., Udeinya, I.J. \& Oligino, L.D. Proc. Natl. Acad. Sci. USA 80, 5075-5079 (1983)

9. Smith, J.D., Subramanian, G., Gamain, B., Baruch, D.I
\& Miller, L.H. Mol. Biochem. Parasitol. 110 293-310 (2000).

10. Smith, J.D. et al. Cel/ 82, 101-110 (1995).

11. Fried, M. \& Duffy, P.E. Science 272, 1502-1504 (1996).

12. Fried, M., Nosten, F., Brockman, A., Brabin, B.J. \& Duffy, P.E. Nature 395, 851-852 (1998).

13. Salanti, A. et al. Mol. Microbiol. 49, 179-191 (2003)

14. Viebig, N.K. et al. EMBO Rep. 6, 775-781 (2005).

15. Viebig, N.K. et al. PLoS ONE 2, e910 (2007).

16. Gamain, B. et al. J. Infect. Dis. 191, 1010-1013 (2005).

17. Salanti, A. et al. J. Exp. Med. 200, 1197-1203 (2004).

18. Tolia, N.H., Enemark, E.J., Sim, B.K. \& Joshua-Tor, L. Cell 122, 183-193 (2005).

19. Singh, S.K., Hora, R., Belrhali, H., Chitnis, C.E. \& Sharma, A. Nature 439, 741-744 (2006).

20. Tan, K. et al. Structure 14, 33-42 (2006).

\title{
A new, leaner and meaner bacterial organelle
}

\author{
Sabine Heinhorst \& Gordon C Cannon
}

\section{Bacteria sequester key metabolic steps into polyhedral protein compartments. A newly discovered nanocompartment reveals what it takes for its cargo protein to become encapsulated within a protein shell.}

Contrary to the long-held belief that prokaryotic cells are essentially unstructured sacks of cytoplasm and that compartmentalization is a prerogative of eukaryotes, recent studies using EM, structural and biochemical approaches have shifted this paradigm. The advances in the field are documented in two recent monographs entirely devoted to the multitude of intracellular structures found in prokaryotes ${ }^{1,2}$, ranging from simple storage granules and cytoskeletal elements to more elaborate structures with key functions in metabolism. Although lipid membrane-enclosed intracellular compartments that qualify as organelles sensu stricto are not common in prokaryotes, some of the complex macromolecular assemblies found in bacteria definitely function in a manner analogous to eukaryotic organelles, allowing their hosts to inhabit certain metabolic and/or geophysical niches.

Particularly intriguing bacterial inclusions are the protein microcompartments-thin polyhedral protein shells containing enzymes that catalyze key steps in bacterial metabolism. By far, the best characterized among these is

Sabine Heinhorst and Gordon C. Cannon are at The University of Southern Mississippi, 118 College Drive, \# 5043, Hattiesburg, Mississippi 39406-0001, USA. e-mail: sabine.heinhorst@usm.edu or gordon.cannon@usm.edu.

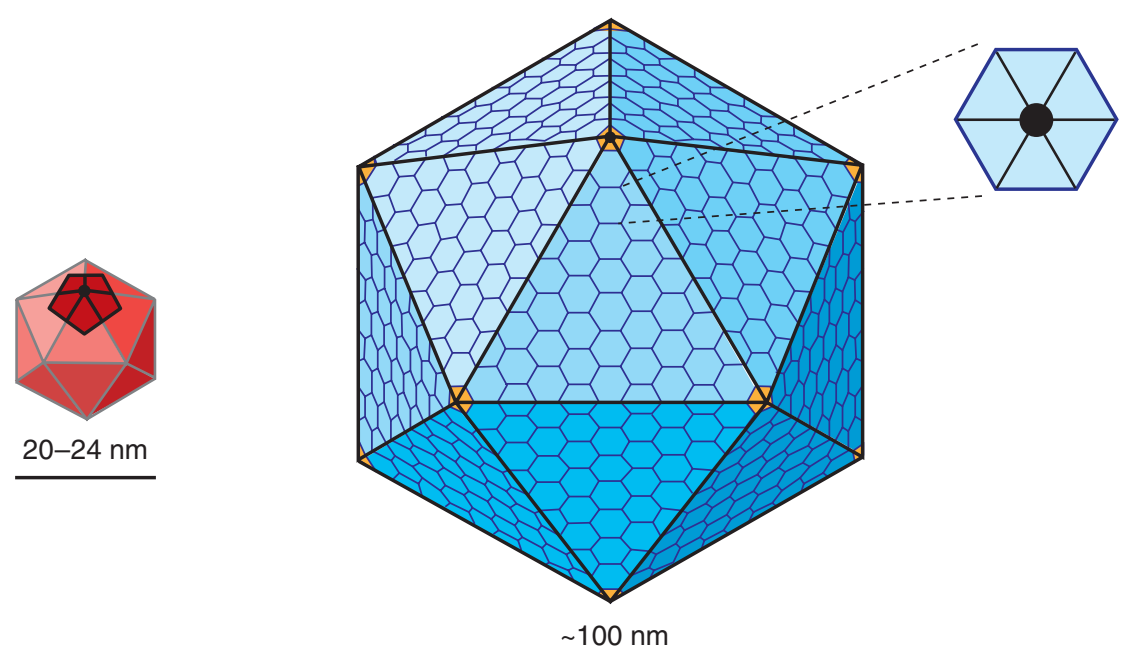

Figure 1 Bacterial nano- and microcompartments. Left, a representation of the newly described icosahedral nanocompartment from T. maritima, whose shell is assembled from 12 pentamers of the protein encapsulin (dark red). Small pores are present in the center of the pentamers, as well as between the monomers and where pentamers meet (not shown). Right, a diagram representing a carboxysome, the prototype bacterial microcompartment. The facets of its shells are made of one type of protein (see enlarged blue hexamer), whereas the vertices are formed by pentamers of another type of protein (yellow); small pores are present in the center of each hexamer and pentamer ${ }^{8}$.

the carboxysome of autotrophic bacteria, which contains the enzyme ribulose 1,5bisphosphate carboxylase/oxygenase (RuBisCO) and is crucial for efficient carbon fixation and bacterial growth at ambient $\mathrm{CO}_{2}$ concentrations ${ }^{3}$. Some heterotrophic bacteria, such as Salmonella enterica, Escherichia coli and Lactobacillus reuteri, form similar polyhedral protein structures that harbor enzymes involved in the catabolism of specialty carbon sources ${ }^{4,5}$. All of these microcompartment shells apparently share a common design principle: the facets are composed of tightly packed hexamers of one protein type, whereas the vertices of the polyhedra are 